\title{
A case of vasospastic angina. Vasospasm physiopathology: a new therapeutic role for ranolazine?
}

\author{
Carlo Uran, Giacomo Di Chiara, Biagio Bosco, Domenico D’Andrea, Pietro Iodice \\ Complex Operative Unit of Cardiology, Intensive Unit Coronary Care, Hospital Centre San Giuseppe e Melorio, ASL \\ Caserta, Italy
}

\begin{abstract}
We report the case of a 40 years-old man, transferred from another hospital to our Intensive Care Unit because of acute coronary syndrome. Coronarography did not show coronary stenosis. 24-hours monitoring EKG allowed diagnosis of Prinzmetal angina and appropriate therapy was administered. Six months after discharge, due to recurrence of symptoms, ranolazine was added to the therapy. After one year patient is symptoms free.
\end{abstract}

Correspondence: Carlo Uran, Complex Operative Unit of Cardiology, Intensive Unit Coronary Care, Hospital Centre San Giuseppe e Melorio, ASL Caserta, Italy.

E-mail: carlura@libero.it

Key words: MINOCA; vasospastic angina; ranolazine.

Conflict of interest: The authors declare no conflict of interest.

Funding: None.

Acknowledgments: Antonella De Nicola is warmly thanked for language revision.

Availability of data and materials: All data underlying the findings are fully available.

Ethics approval and consent to participate: No ethical committee approval was required for this case report by the Department, because this article does not contain any studies with human participants or animals. Informed consent was obtained from the patient included in this study.

Consent for publication: The patient gave his written consent to use his personal data for the publication of this case report and any accompanying images.

Received for publication: 4 April 2020.

Accepted for publication: 10 June 2020.

${ }^{\circ}$ Copyright: the Author(s), 2020

Licensee PAGEPress, Italy

Monaldi Archives for Chest Disease 2020; 90:1295

doi: 10.4081/monaldi.2020.1295

This article is distributed under the terms of the Creative Commons Attribution Noncommercial License (by-nc 4.0) which permits any noncommercial use, distribution, and reproduction in any medium, provided the original author(s) and source are credited.

\section{Introduction}

Incidence of myocardial infarction or ischemia with nonobstructive coronary arteries (MINOCA/INOCA) is increasing. The majority of these patients has failure of coronary microvascular function, elevated risk of cardiovascular events and is prone to suffering from heart failure with preserved ejection fraction. The definitions of MINOCA/INOCA include a set of syndromes defined as myocardial infarction or ischemia with non-obstructive coronary arteries, or presence of plaque involving $50 \%$ or less of coronary lumen, with incidence of almost $65 \%$ in women and $32 \%$ in men. Epicardial causes of MINOCA include coronary plaque disruption, coronary dissection, and coronary spasm. Microvascular MINOCA mechanisms involve microvascular coronary spasm, Takotsubo syndrome, myocarditis, and coronary thromboembolism. Underlying mechanisms of these clinical features are multifactorial and can operate alone or by cooperation. A peculiar MINOCA outset can be the vasospastic or variant angina (Prinzmetal angina) [1]. The variant angina described by Prinzmetal in 1959 [2] is a clinical entity characterized by chest pain at rest with transient ST segment elevation at EKG, with a prompt response to nitrates. Coronary spasm may develop after exposure to cold water, substances that promote vasoconstriction as alpha agonists (pseudoephedrine, oxymetazoline), parasympathomimetic agents (methacholine), recreational drugs (cocaine), prolonged mental stress and hyperventilation, or beta-blockers [2-3]. The incidence of vasospastic angina is unknown and its prevalence depends on the practice to investigate it by provocative tests. This is the reason why coronary spasm may be more frequent than reported in current literature [4]. Classical therapy is based on the use of calcium channel blockers, nitrates and statin. Ranolazine, anti-angina drug recommended in chronic stable angina, is rarely prescribed or not used at all. We hypothesized a possible therapeutic role of ranolazine.

\section{Case Report}

An obese 40 years-old man was transferred to our ICU from another hospital, where he came referring chest pain, dizziness and dyspnea. EKG was normal and blood chemistries pointed out a slight increase in Troponin I. Diagnosis of non-ST segment elevation myocardial infarction (NSTEMI) was made at that time. In our ICU, patient was symptoms free and reported a family history of diabetes. He was a smoker ( 5 cigarettes/day), he referred hypertension but was actually not on treatment (he had been on treatment with dihydropyridine calcium channel blockers, withdrawn because of ankles edema). He had neither diabetes nor dyslipidemia. No 
fever was reported as well. BMI and vital parameters were checked, venous sampling for blood chemistry test was performed and Global Registry of Acute Coronary Events (GRACE) score was calculated (Table 1). EKG (Figure 1a), echocardiogram and thorax X-ray were normal. We excluded myocarditis because of normality on inflammatory indexes and patient anamnestic reports. A therapy with metoprolol (25 mg bid); ASA (100 mg/die), Atorvastatin ( $80 \mathrm{mg} /$ day); enoxeparin $100 \mathrm{U} / \mathrm{Kg}$ bid and aspirin (100 mg/day) was prescribed. Because of low risk of cardiovascular events at six months, calculated by Grace score, after 36 hours a coronarography was performed and a non-obstructive coronary was found. (Figure $1 \mathrm{~b}$ ). Diagnosis of MINOCA was made and Ticagrelor (90 $\mathrm{mg}$ bid) was added to therapy. After 72 hours blood values of cardiac enzymes were returned to normal, sinus bradycardia (40 bpm) was displayed at EKG and patient, free of symptoms, was transferred to post-ICU room. Holter monitoring was performed in order to check the cardiac rhythm. At 06:15 in the morning (after 20 hours of monitoring), the nurse was called by the patient who referred chest pain and dizziness, spontaneously solved after 2 minutes. Holter monitoring was immediately developed, so that ST up in the inferior leads, and sustained ventricular tachycardia were displayed (Figure 2). Due to the suspect of vasospastic angina, the patient was asked to undergo the Cath lab to perform a coronarography again, within provocative tests, in order to confirm such diagnosis. Patient refused the tests and therapy was empirically modified, discontinuing metoprolol, ticagrelor, aspirin and enoxeparin. diltiazem $60 \mathrm{mg}$ tid was added. Patient was under observation for another 48 hours, and discharged on the sixth day with the following diagnosis: Prinzmetal angina, complicated with sustained ventricular tachycardia. The following therapy was prescribed: diltiazem $60 \mathrm{mg}$ tid, nitroglycerin TS $10 \mathrm{mg}$ (from $9 \mathrm{pm}$ to $4 \mathrm{pm}$ ); atorvastatin $80 \mathrm{mg}$ and indication to undergo cardiac MRI that patient brought into view after one month from discharge, MRI displayed, in T2-weighted sequences, small areas of post-ischemic edema in the right coronary field. At the three months follow up, patient lost some weight, was symptoms free and, another cardiac MRI performed, showed disappearance of those ischemic areas. After six months of full wellness, patient returned to our observation referring short lasting chest pain at rest, accompanied by mild dyspnea. Blood pressure was normal (120/70 mmHg), EKG displayed a sinus bradycardia $(50 \mathrm{bpm})$; echocardiogram displayed an EF of $60 \%$. Blood chemistry tests did not show noteworthy changes as well. Patient reported he had never stopped the prescribed therapy and so ranolazine ( $375 \mathrm{mg}$ bid) was added too Moreover, on such occasion we proposed to the patient a cardiac MRI but unfortunately he refused it. At one year follow-up, patient is completely symptom free. A cardiac MRI has been proposed again but the patient refused to undergo it.

\section{Physiopathology and Discussion}

A recent Italian study which evaluated patients suffering from myocardial infarction with non-obstructive coronary arteries,

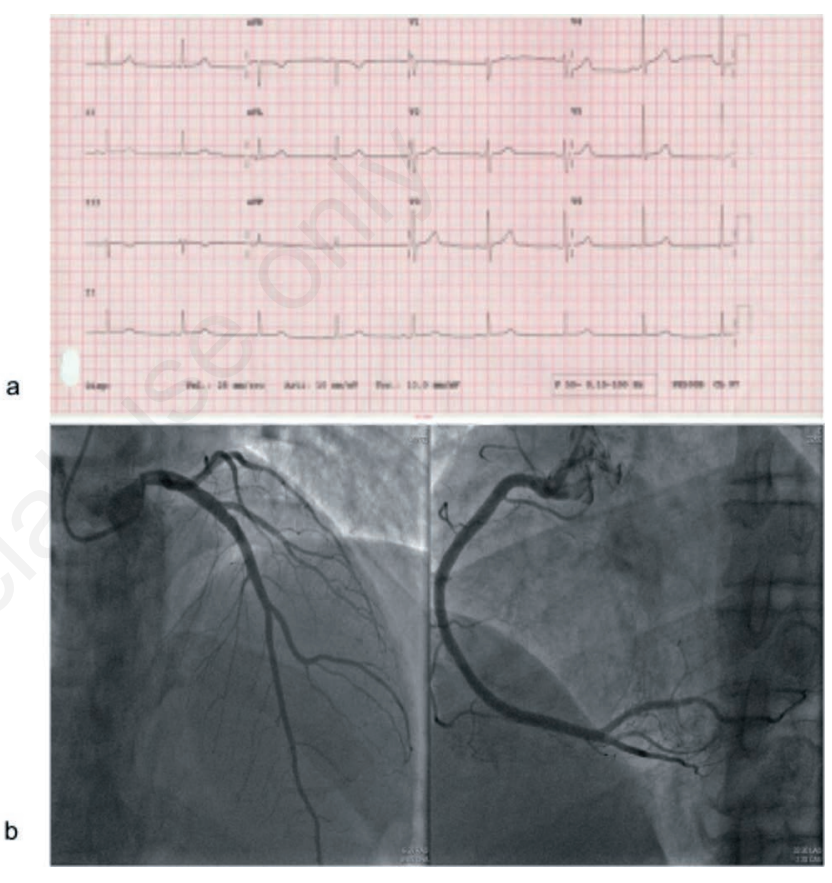

Figure 1. a) First EKG. b) Coronarography.

Table 1. Laboratory tests and vital parameters.

\begin{tabular}{|c|c|c|c|c|c|c|c|c|c|}
\hline & Normal vaules & Charge & 6 hours & 12 hours & 24 hours & Day 2 & Day 3 & Day 4 & Discharge \\
\hline BMI & $18.50-24.99$ & 32.51 & & & & & & & 31.56 \\
\hline Blood Pressure (mm/Hh) & & $130 / 90$ & & & & & & & $120 / 70$ \\
\hline Breath frequency Acts/min & $12-16$ & 20 & & & & & & & 18 \\
\hline Troponin (ng/mL) & $0.00-0.05$ & 0.19 & 0.44 & 0.67 & 0.34 & 0.2 & 0.17 & 0.1 & 0.05 \\
\hline CK-MB U/L & $0.00-6.3$ & 3.2 & 4.3 & 7.1 & 5.2 & 4.3 & 3.3 & & 3.2 \\
\hline RCP mg/dL & $0.00-0.5$ & 0.4 & & & & & & & 0.3 \\
\hline Glycemia mg/dL & $80-100$ & 133 & & & & & & & 110 \\
\hline Uricaemia mg/dL & & 7 & & & & & & & 6 \\
\hline Total cholesterol mg/dL & $30-200$ & 207 & & & & & & & 200 \\
\hline LDL mg/dL & $<135$ & 135 & & & & & & & 132 \\
\hline HDL mg/dL & $35-120$ & 35 & & & & & & & 37 \\
\hline $\mathrm{EF} \%$ & $55-60$ & & & & & & & & $55-60$ \\
\hline $\mathrm{SpO}_{2}$ & $>96 \%$ & $98 \%$ & & & & & & & $98 \%$ \\
\hline GRACE risk score & & 37 & & & & & & & \\
\hline
\end{tabular}


demonstrated induction of coronary artery spasm in about $46 \%$ of cases [5]. The pathogenesis of coronary artery spasm is not completely clarified and different pathogenic mechanisms have been proposed. Many evidences indicate that small guanosine triphosphatase RhoA and its effector Rho-kinase are involved in the regulation of vascular muscle cell contractility. The RhoA/Rho-kinase pathway, through inhibition of myosin-light-chain-phosphatase, promotes an increase of intracellular calcium and leads to calcium sensibilization in response to vasoconstrictor stimuli. It explains the therapeutic effectiveness of calcium channel blockers in suppressing coronary spasm. Rho-kinase promotes cardiovascular disease by enhancing the production of reactive oxygen species too [6], leading to an increasing of the oxidative stress, involved in the pathogenesis of vasospasm as well [7]. The endothelium plays a fundamental role in regulation of coronary tone by releasing endothelium-derived relaxing factors as vasodilator prostaglandins, nitric oxide (NO), and endothelium derived hyperpolarizing factor. So the decrease of NO release due the endothelial dysfunction, combined with vascular muscular cell hyperreactivity, may have a crucial role in coronary spasm. By contrast, a porcine model of spasm indicates that endothelial function is preserved at the site of spasm. So, a decrease in the releasing of NO has a role in the pathogenesis of vasospasm, but the endothelial disfunction alone may not be sufficient to explain the vasospasm itself [8]. In patients with vasospastic angina, chronic low-grade inflammation may be demonstrated by increased CRP, monocyte chemoattractant protein-1 and interleukin-6. A study in patients with and without vasospastic angina, using $18 \mathrm{~F}$-fluorodeoxyglucose positron emission tomography/computed tomography, demonstrated that coronary spasm was associated with increase of $18 \mathrm{~F}$-fluorodeoxyglucose uptake in coronary adventitia and perivascular adipose tissue [9]. A crucial role has the autonomic nervous system too. Enhanced sympathetic or parasympathetic nervous activity and sympathovagal imbalance may have a strong role in vasospastic angina [10].

\section{About this case}

Patient clinical picture made us suspect a NSTEMI. Due to low risk mortality at six months calculated by GRACE score [11] (less than $1 \%$ ), coronarography was performed after 36 hours from charge, without provocative tests, because of lack of any vasospastic angina suspect. Recently, diagnostic criteria of vasospastic angina have been published by Coronary Vasomotor Disorders International Study group (COVADIS) group: symptoms solved by nitrates intake; proved myocardial ischemia during episodes; angiographic demonstration of coronary spasm. All the three criteria must be present to make a definite diagnosis. Most frequent EKG changes during a coronary spasm are the appearance of a peaked and symmetrical T wave (50\% of cases); ST-segment elevation or depression; $\mathrm{T}$ wave negativization; increase in the height and width of the $\mathrm{R}$ wave and a coincident decrease in the magnitude or disappearance of the $\mathrm{S}$ wave. A negative $\mathrm{U}$ wave can appear too. Many forms of arrhythmia can appear during vasospasm: ventricular tachycardia, ventricular fibrillation, supraventricular tachyarrhythmias and any degree of AV block as well. The longer an episode lasts, the higher is the prevalence and importance of ventricular arrhythmias. Fundamental step in confirming the diagnosis is vasospam induction, during cardiac catheterization, spontaneously or by pharmacological or non pharmacological tests (ergonovine, acetylcholine, hyperventilation). These tests are warranted only when diagnosis of vasospastic angina is suspected but not firmly established. A positive provocative test for coronary vasospasm must induce all of the following: reproduction of the usual chest pain, ischaemic electrocardiogram changes, and $\geq 90 \%$ vasoconstriction on angiography [12]. In our opinion, this case is worth of interest for two reasons: changes in EKG observed in real time and efficacy of ranolazine. Concerning therapy, as reported in several studies, we prescribed calcium channel blocker and statin. Either dihydropyridine or non dihydropiri-
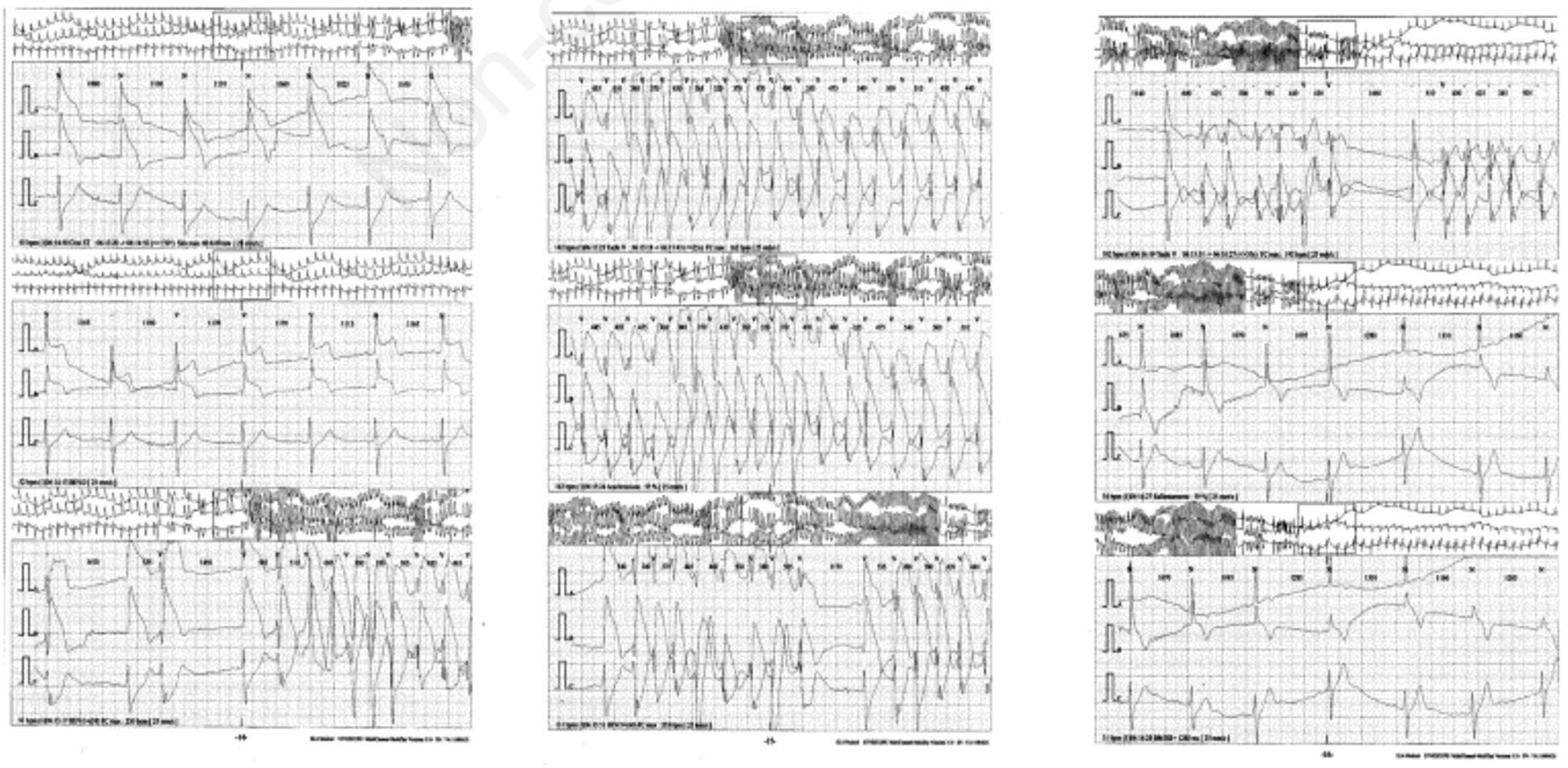

Figure 2. Holter monitoring strips. 
dine can be used, depending on patient characteristics, the combination of both may be useful as well, but there are few data about this approach. Several studies have shown that statins (fluvastatin, atorvastatin and rosuvastatin) are effective at preventing coronary artery vasospasm [13]. Aspirin was discontinued because its use is controversial and there are lack of evidences about its benefic effects in vasospastic angina: its intake might lead to higher incidence for rehospitalization in such kind of patients as well [14]. Nitrates in cooperation with calcium channel blocker, reduce frequency of symptoms in patients with vasospastic angina. In our case symptom arose at 06:15 a.m., so, against the guidelines that recommend their use in combination with calcium channel blockers only in case patients are symptomatic yet [15], we added nitrate transdermal to cover night and morning hours. Despite therapy, after six months patient was symptomatic again. Diltiazem dosage was not increased because of the cardiac frequency of $50 \mathrm{bpm}$, neither a combination with dihydropiridinic calcium channel blocker was made, because of anamnestic side effects referred by patient. For those reasons we decided to add ranolazine $375 \mathrm{mg}$ bid. We used a low-dose because ranolazine is extensively metabolized by cytochrome P450(CYP)3 enzymes and so increases HMG-CoA reductase inhibitor and calcium channel blockers activity as well.

\section{Conclusions}

Our case is interesting because of difficulty documenting, off the Cath-lab, where provocative test can be performed, symptoms, changes in EKG and incidental ventricular arrythmia as well. That is the reason why the vasospasm should always be investigated, at the moment of coronarography, by provocative tests, in patients with non-obstructive coronary arteries. The efficacy of ranolazine is interesting above all. In our opinion, in fact, ranolazine deserves a special attention. This drug is a piperazine derivative, approved as antianginal in stable coronary artery disease. It acts by blocking the late $\mathrm{Na}+$ current $\left(\mathrm{INa}^{+}\right)$, so prevents the rise of cytosolic calcium and decreases myocardial wall tension, improving coronary blood flow. Ranolazine has some anti-glycemic effects as well. In fact, it has shown a reduction of hemoglobin A1c in diabetic patients suffering from chronic stable angina [16]. In patients suffering from peripheral arterial disease, ranolazine showed an increase in peak walking time in comparison to placebo. This may be secondary to an $\alpha 1$ - adrenergic receptor blocking effect, without altering heart rate or systemic blood pressure [17]. Ranolazine in pulmonary hypertension patients showed improvement in functional class, in RV function and in exercise time. Nevertheless, no improvement was showed in hemodynamic parameters [18]. A recent study showed increased levels of adenosine, a potent dilator of resistance coronary vessel, in murine endothelial cells and cardiomyocite incubated with ranolazine [19]. At last, a small study shows ranolazine improves angina in women with non-obstructive coronary [20]. In summary, given ranolazine mechanism of action, its effect on adenosine, its effectiveness in some arterial diseases, given our record and the almost lack of literature data, we suggest a randomized trial, to show any ranolazine benefits in vasospastic angina treatment.

\section{References}

1. Pacheco Claudio C, Quesada O, Pepine CJ, Noel Bairey Merz C. Why names matter for women: MINOCA/INOCA (myocar- dial infarction/ischemia and no obstructive coronary artery disease) Clin Cardiol 2018;41:185-93.

2. Prinzmetal M, Kennamer R, Merliss R, Wada T, Bor N: Angine pectoris. I. A variant form angine pectoris. A preliminar report. Am J Med 1959;27:375-88.

3. Turlapaty PD, Altura BM. Propranolol induces contractions of canine small and large coronary arteries. Basic Res Cardiol 1982;77:68-81.

4. Picard F, Sayah N, Spagnoli V, et al. Vasospasm angina; A literature review of current evidence Arch Cardiovasc Dis 2019;112:44-55.

5. Montone RA, Niccoli G, Fracassi F, et al. Patients with acute myocardial infarction and non-obstructive coronary arteries: safety and prognostic relevance of invasive coronary provocative tests. Eur Heart J 2018;39:91-8.

6. Shimokawa H, Sunamura S, Satoh K. RhoA/Rho-Kinase in the Cardiovascular System. Circ Res 2016;118:352-66.

7. Miyamoto S, Kawano H, Sakamoto T, et al. Increased plasma levels of thioredoxin in patients with coronary spastic angina. Antioxid Redox Signal 2004:6;75-80.

8. Miyata K., Shimokawa H, Yamawaki T, et al. Endothelial vasodilator function is preserved at the spastic/inflammatory coronary lesions in pigs. Circulation 1999;100: 1432-7.

9. Ohyama K, Matsumoto Y, Takanami K, et al. Coronary adventitial and perivascular adipose tissue inflammation in patients with vasospastic angina. J Am Coll Cardiol 2018;71; 414-25.

10. Miwa K, Igawa A, Miyagi Y, et al. Alterations of autonomic nervous activity preceding nocturnal variant angina: sympathetic augmentation with parasympathetic impairment. Am Heart J 1998;135;762-71.

11. Eagle KA, Lim MJ, Dabbous OH, et al. A valided prediction model for all forms of acute coronary syndrome: estimating the risck of 6-month postdischarge death in an international registry. JAMA 2004;221:2727-33.

12. Beltrame JF, Crea F, Kaski KC, et al. International standardization of diagnostic criteria for vasospastic angina. Eur Heart J 2017;38;2565-8.

13. Ishii M, Kaikita K, Sato K, et al. Impact of Statin Therapy on Clinical Outcome in Patients With Coronary Spasm. J Am Heart Assoc 2016;5:e003426.

14. Lim AY, Park TK, Cho SW et al. Clinical implications of lowdose Aspirin on vasospastic angina patients without significant coronary artery stenosis; a propensity score matched analysis. Int J Cardiol 2016;221:161-6.

15. Roffi M, Patrono C, Collet JP, et al. 2015 ESC Guidelines for the management of acute coronary syndromes in patients presenting without persistent ST-segment elevation: Task Force for the Management of Acute Coronary Syndromes in Patients Presenting without Persistent ST-Segment Elevation of the European Society of Cardiology (ESC). Eur Heart J 2016;37:267-315

16. Chaitman BR, Pepine CJ, Parker JO, et al. Combination Assessment of Ranolazine In Stable Angina (CARISA) Investigators. Effects of ranolazine with atenolol, amlodipine, or diltiazem on exercise tolerance and angina frequency in patients with severe chronic angina: a randomized controlled trial. JAMA 2004;291:309-16.

17. Supervised Treadmill Exercise and Ranolazine for Intermittent Claudication of Lower Extremities (STERILE, NCT00914316). Available from: https://www.clinicaltrials.gov/ct2/show/ NCT00914316. 
18. Han Y, Forfia PR, Vaidya A, et al. Rationale and design of the ranolazine PH-RV study: a multicentred randomised and placebo-controlled study of ranolazine to improve RV function in patients with non-group 2 pulmonary hypertension. Open Heart 2018;5:e000736.

19. Le DE, Davis CM, Wei K, et al. Ranolazine may exert its ben- eficial effects by increasing myocardial adenosine levels. Am J Physiol Heart Circ Physiol 2020;318:H189-H202.

20. Mehta PK, Goykhman P, Thomson LEJ, et al. Ranolazine improves angina in women with evidence of myocardial ischemia but no obstructive coronary artery disease. JACC Cardiovasc Imaging 2011;4:514-22. 